\title{
Electric field sensing and imaging by noninvasive parallel-plate sensor
}

\author{
Masahiro Tsuchiya $^{1 \mathrm{a})}$, Takahiro Shiozawa ${ }^{2}$, \\ and Shinji Harakawa ${ }^{3,4}$ \\ ${ }^{1}$ Institute of Information and Communications Technology, \\ 4-2-1 Nukui-kitamachi, Koganei, Tokyo 184-8795, Japan \\ 2 Department of Communication Network Engineering, \\ National Institute of Technology, Kagawa College, \\ 551 Kohda, Takuma, Mitoyo, Kagawa 769-1192, Japan \\ ${ }^{3}$ Hakuju Institute for Health Science Co. Ltd., \\ 1-37-5 Tomigaya, Shibuya-ku, Tokyo 151-0063, Japan \\ ${ }^{4}$ Obihiro University of Agriculture and Veterinary Medicine, \\ Inada-cho, Obihiro, Hokkaido 080-8555, Japan
}

a)mtsu@nict.go.jp

\begin{abstract}
An innovative concept of noninvasive sensor device has been proposed and successfully demonstrated, which makes direction detections and intensity measurements for electric fields be drastically simplified and agile. Its primal feature is a unique geometry, with the simplicity of Columbus' egg, where an ammeter and optical readout are internally contained in virtual-shorted parallel plates. While its electromagnetic principle for accurate sensing has been confirmed, its function to promptly image directions and distributions of electric fields has been demonstrated combining its aerial scan with a bulb-shuttered photographic camera.
\end{abstract}

Keywords: dipole potential, electric field sensor, electromagnetic design theory, imaging, noninvasiveness, optical readout

Classification: Electronic instrumentation and control

\section{References}

[1] For example, R. A. Millikan: Phys. Rev. 39 (1932) 397. DOI:10.1103/PhysRev.39. 397

[2] For example, S. G. Gathman and R. V. Anderson: Rev. Sci. Instrum. 36 (1965) 1490. Also references therein. DOI:10.1063/1.1719364

[3] P. S. Maruvada, R. D. Dallaire and R. Pedneault: IEEE Trans. Power Apparatus Syst PAS-102 (1983) 738. DOI:10.1109/TPAS.1983.318035

[4] H. I. Bassen and G. S. Smith: IEEE Trans. Antenn. Propag. AP-31 (1983) 710. DOI:10.1109/TAP.1983.1143126

[5] T. P. Budka, S. D. Waclawik and G. M. Rebeiz: IEEE Trans. Microw. Theory Tech. 44 (1996) 2174. DOI:10.1109/22.556445

[6] G. A. Massey, D. C. Erickson and R. A. Kadlec: Appl. Opt. 14 (1975) 2712. DOI:10.1364/AO.14.002712

[7] J. A. Valdmanis, G. Mourou and C. W. Gabel: Appl. Phys. Lett. 41 (1982) 211. 
DOI:10.1063/1.93485

[8] T. Ohara, M. Abe, S. Wakana, M. Kishi and M. Tsuchiya: Tech. Digest 1st JapanKorea Joint Workshop Microw. Photon. (2000) 66.

[9] M. Tsuchiya and T. Shiozawa: IEEE Photonics Society Newsletter 26 [6] (2012) 9.

[10] LEI camera web site: http://lei-camera.nict.go.jp.

[11] For example, H. B. Palmer: Electr. Eng. 56 (1937) 363. DOI:10.1109/EE.1937. 6540485

[12] W. K. H. Panofsky and M. Phillips: Classical Electricity and Magnetism (Addison-Wesley, Reading, 1978) 2nd ed. 8.

[13] R. Justice and V. H. Rumsey: IRE Trans. Antennas Propag. 3 (1955) 177. DOI:10. 1109/TAP.1955.1144315

[14] W. K. H. Panofsky and M. Phillips: Classical Electricity and Magnetism (Addison-Wesley, Reading, 1978) 2nd ed. 14.

[15] For example, W. E. Kock and F. K. Harvey: Bell Syst. Tech. J. 30 (1951) 564. DOI:10.1002/j.1538-7305.1951.tb03670.x

\section{Introduction}

Electric field is invisible although it exists not only in and around almost every technological equipment and facility but also in a wide variety of circumstances in our everyday lives. The invisibleness prevents us, except a limited number of experts, from easily imagining or being sensitive to its behaviors. If our apprehension of electric field and related electrical phenomena could be enhanced through a simple, agile and easily available approach with a reasonable accuracy, its resultant impacts would be immeasurable from technology, education and daily living habits points of views. Furthermore, the impacts would be multiplied if a technique to image electric field behaviors with similar degrees of simplicity, agility, accuracy, and availability could be established.

Besides electroscopes [1] and their posterity, conventional techniques to sense electric field are categorized into four groups: field meters [2, 3], antennas [4], electrical probes [5], and electrooptic (EO) approaches $[6,7,8,9,10]$. The first and second are well-established but cumbersome, where impeded are the abovementioned simplicity, agility, and availability. The third could be considerably compact whereas its accuracies are not always satisfactory. A major drawback in these three categories is the need for measuring instruments additionally to their transducers and eventually their connecting cables, which prevents the noninvasiveness and resultant sensing accuracy in a personal-area or finer measurement. The situation for the electroscopes is similar.

The last category is free from the cable issue and, therefore, has been the trend of recent decades, which is superior also in the high speed properties [7]. Additional features of spatial resolutions in the micron range together with moderate agility have been introduced by the optical fiber edge concept [8]. Furthermore, the function of real-time visualization has been recently realized by the live electrooptic imaging technique $[9,10]$, providing an ultimate style of intuition. However, it should be emphasized that the above-mentioned goal of simplicity, agility, and availability is far beyond the EO approaches, yet. 
Newly proposed in this paper is a novel concept of electric field sensor, which innovatively satisfies all the above requirements. Its commercial availability should be a future trial but seems promising owing to its extreme simplicity. Described below are not only successful results of its proof-of-principle experiments but also electric field images derived promptly for an everyday gear, an external hard disk drive (HDD) unit, suggesting its substantial potential in such practical applications as assessments of personal environments.

\section{Electromagnetic concept of the sensor}

The sensor concept originates from an electromagnetic consideration on a rather classical parallel-plate capacitor [11] depicted in Fig. 1(a). Its internal electric field $\mathbf{E}_{\text {rms }}$ is often assumed to be uniform, where its absolute value $E_{\mathrm{rms}}$ is given by Eq. (1) via the Gauss's flux theorem [12]:

$$
E_{\mathrm{rms}}=I_{\mathrm{rms}} /\left(2 \pi f \varepsilon_{0} S\right),
$$

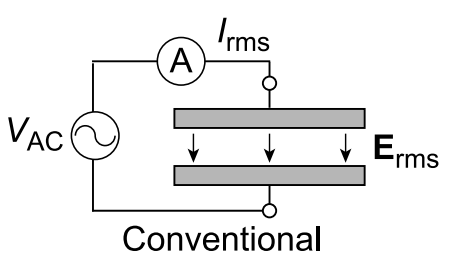

(a)

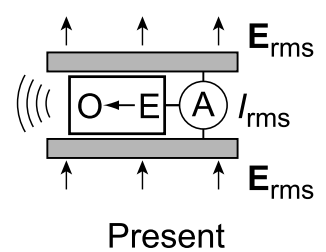

(c)

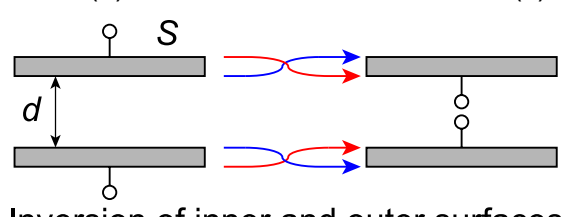

(b)

Fig. 1. Sensor device named electric field imager (EFIM) is conceptually illustrated. (a) Classical parallel-plate capacitor. (b) Geometric inversion of inner and outer surfaces of the plates. (c) EFIM configuration equipped with an optical readout. E: electrical, O: optical.

where $I_{\text {rms }}, f, \varepsilon_{0}$, and $S$ are the current measured by an external ammeter, the electric field frequency, the permittivity of vacuum, and the plate area, respectively. The following equation with time $t$ should be used for the DC case:

$$
E_{\mathrm{DC}}=\int_{0}^{t} I_{\mathrm{DC}} d t / \varepsilon_{0} S
$$

while the AC case is assumed for Fig. 1, Eq. (1) and hereafter. Let the capacitor exterior be turned inside as schematically shown in Fig. 1(b): each plate is flipped and the ammeter is embedded in between as shown in Fig. 1(c). Suppose an external electric field $\mathbf{E}_{\text {rms }}$ having a sinusoidal temporal waveform, which is applied uniformly and perpendicularly to the parallel plates. Here, it should be noted that the behaviors of electrical charges in the parallel plates are analogous to those in an ungrounded thin metal plate in the same circumstance. As upward $\mathbf{E}_{\text {rms }}$ increases from zero with time, polarization arises within the thin metal plate in accordance 
with the Gauss's flux theorem, where positive charges accumulating on its upper surface originate $\mathbf{E}_{\mathrm{rms}}$ and negative charges on its lower surface terminate $\mathbf{E}_{\mathrm{rms}}$. The polarization alternates in accordance with $\mathbf{E}_{\mathrm{rms}}$ and internal alternating charge flows, i.e., AC current, are generated. In the case of the parallel plates in Fig. 1(c), the AC current $I_{\mathrm{rms}}$ is measured by the ammeter and the same relationship between $\mathbf{E}_{\text {rms }}$ and $I_{\text {rms }}$ as Eq. (1) is given. The external electric field is thus evaluated by the measured internal current. In addition, the embedment of the ammeter and conducting wires is indispensable since their invasiveness to the sensing and resultant measurement inaccuracy are suppressed as mentioned below. This is the principle of the present electric field sensing, which has never been reported previously, to the best of the authors' knowledge. Requirements for its satisfactory noninvasiveness and resultant accuracy are listed below.

The first requirement is given by a shape factor that is the same as the wellknown assumption for the electric field uniformity in Fig. 1(a): the ratio of the plate breadth $\sim \sqrt{S}$ to the plate distance $d$ should be large enough. In addition, the circuitry should be sufficiently distant from the plate edges to suppress its interaction with $\mathbf{E}_{\mathrm{rms}}$. The second is concerned about the sensor readout. It should be electrically isolated from $\mathbf{E}_{\text {rms }}$, for which wireless approaches are advantageous [13] and an optical readout maximizes the isolation. The third is related to the internal potential difference, i.e., the voltage $V_{\mathrm{pp}}$ between the parallel plates. If the internal impedance of the ammeter could be eliminated, $V_{\mathrm{pp}}$ would be zero and, hence, the parallel plates could simulate a thin metal plate. This is beneficial since noninvasiveness of a tiny thin film is the highest as long as metal materials are used. The virtual short configuration for operational amplifiers is useful for this purpose. It could also eliminate ambiguity of dielectric property between the plates of a finite $d$ value, easing possible calibration.

The fourth requirement is provided by a size factor. Whereas $S$ is dominant for the spatial resolution, important from the noninvasiveness point of view is a volume ratio $V_{\mathrm{e}} / V_{0}$. Here, the volumes $V_{\mathrm{e}}$ and $V_{0}$ are defined by the ranges where electric field disturbance is induced by the sensor insertion and where the original $\mathbf{E}_{\mathrm{rms}}$ is scoped to be uniform, respectively. If $V_{\mathrm{e}} / V_{0}$ is small enough, the noninvasiveness and the accuracy as well as spatial resolution should be satisfactory. While $V_{\mathrm{e}}$ is minimized for a perpendicular electric field [Fig. 1(c)], the volume ratio is still a good index for the sensor angled by $\theta$ [Fig. 2 (a)]:

$$
E_{\mathrm{rms}} \cos \theta=I_{\mathrm{rms}} /\left(2 \pi f \varepsilon_{0} S\right) .
$$

It should be noted that the electric field disturbance near the tilted sensor is caused by the tilt-induced generation of in-plate dipole, which disappears as the dipole potential decreases in proportion to the square of distance [14]. Preliminary numerical simulations suggest that $V_{\mathrm{e}}$ is approximately scaled by the outer plate diameter from the plate edges although more quantitative analyses are needed and will be a future work.

\section{Prototype of electric field imager}

All the four requirements are reasonably satisfied by a prototype shown in Fig. 2(b), which is dedicated for the AC operation and named electric field imager 
(EFIM). As shown schematically in Fig. 2(c), its ammeter output $\alpha I$ in voltage goes to a set of parallel comparators, $i$-th of which lights one of arrayed LEDs in accordance to the $i$-th reference voltage $V_{\mathrm{i}}$. The electric field is thus readout to an experimenter, by whom the number of lit LEDs $N$ is counted. It should be noted that the direction of electric field vector can be detected by rotating the EFIM sensor: $N$ decreases as $\theta$ increases and $\mathbf{E}_{\text {rms }}$ is parallel to the plates at the $N$ minima.

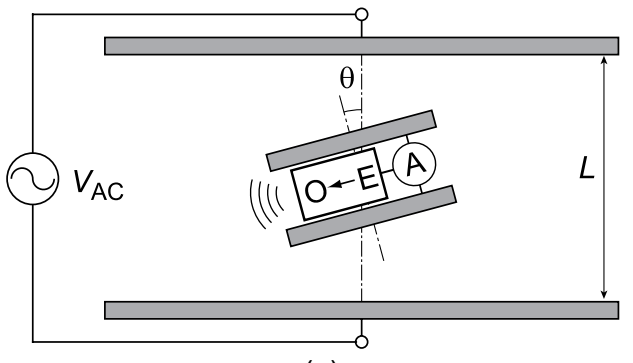

(a)

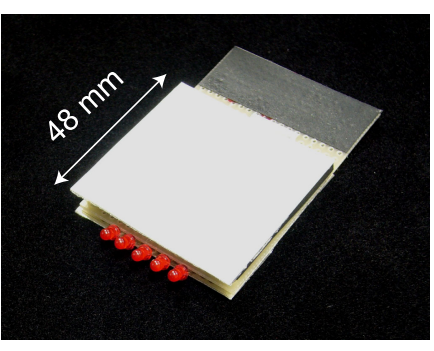

(b)

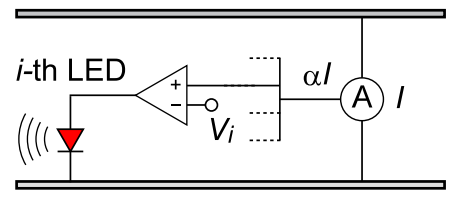

(c)

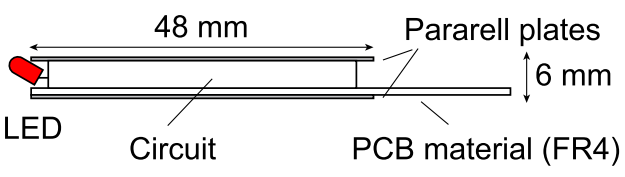

(d)

Fig. 2. (a) Setup for characterization of EFIM sensor. A pair of plate electrodes generates external electric field by AC voltage $V_{\mathrm{AC}}$ of $50 \mathrm{~Hz}$. (b) A photograph of implemented EFIM device. Its circuit diagram is in (c) while its schematic side view is in (d). FR-4: flame retardant type-4, PCB: printed circuit board.

Its side view drawn schematically in Fig. 2(d) indicates two $48 \mathrm{~mm}$ square metal plates, which are covered with insulator films on both surfaces so as to avoid their unintentional electrical contacts to the circuit and objects around them. The circuit together with a battery is implemented on a printed circuit board material of flame retardant type 4, whose one end is stretched outward as a holding part. An array of 5 LEDs is set along an edge of the EFIM sensor, which is intended for higher LED visibility but could degrade the noninvasiveness. Although possible use of transparent electrodes improves the situation, the present configuration was chosen from the rougher-and-ready viewpoint.

To characterize the EFIM sensor, $300 \mathrm{~mm}$ square plates in parallel were kept apart by a distance $L$ of $140 \mathrm{~mm}$ to form an electric field generator, to which $50 \mathrm{~Hz}$ voltage $V_{\mathrm{AC}}$ was applied [Fig. 2(a)]. The EFIM sensor was set horizontally as well as under non-zero $\theta$ conditions by using styrene foam blocks in the center of the generated electric field distribution. Measured results are shown in Fig. 3, where the vertical axes are evaluated by $V_{\mathrm{AC}} / L$ and the red squares indicate the measured thresholds of LED lighting. The linearly aligned red squares in Fig. 3(a) coincide with the design theory (Eq. (1)) except some deviations, which can be ascribed to fluctuated comparator characteristics. The $1 / \cos \theta$ dependence of $E_{\mathrm{rms}}$ needed for four lit LEDs in Fig. 3(b) also agrees with Eq. (3), suggesting the validity of the present dipole potential considerations. 


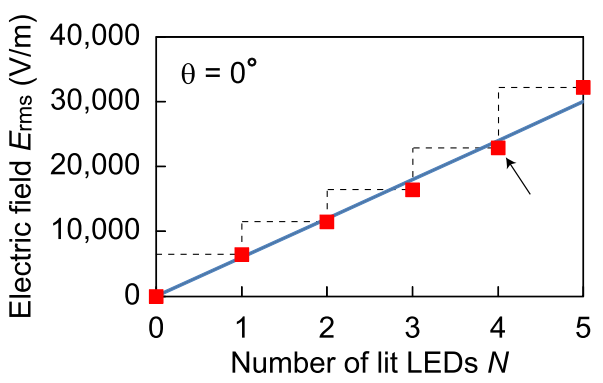

(a)

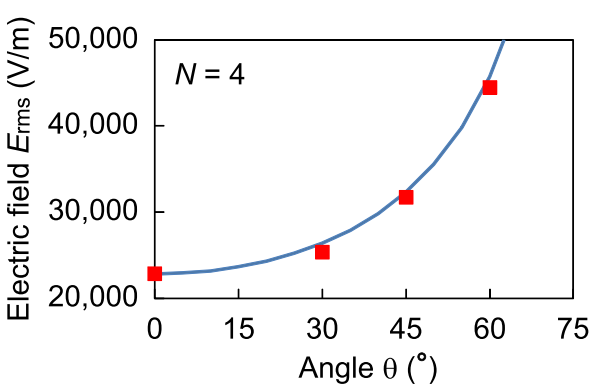

(b)

Fig. 3. Characteristics of the implemented EFIM sensor: (a) applied perpendicular electric field versus the number of lit LEDs, where red squares and dotted lines show thresholds of LED lighting and the trajectory of measurement, respectively. (b) Applied field needed to light the fourth LED, i.e., the condition indicated by an arrow in (a), against the EFIM sensor angle with respect to the field generator electrodes. In (a) and (b), respective blue curves represent Eqs. (1) and (3), assuming that the LED thresholds are proportional to the corresponding internal current values.

\section{Imaging experiments}

Agile electric field visualizations were demonstrated as shown in Fig. 4, where concerned is evanescent electric field over an external HDD unit [Fig. 4(a)]. The imaging setup consists of the EFIM sensor and a photographic camera: the former is spatially scanned by hand with a constant velocity, where its LED array faces the latter under a bulb shutter condition [15] with no lighting and an ND-4 filter attached for the sensitivity adjustment. The EFIM sensor was held at an end of sufficiently long insulating rod so as to avoid unnecessary influence of the experimenter at the other end on $\mathbf{E}_{\text {rms. }}$. No LED was lit without HDD power supply.

Shown in Figs. 4(b) and 4(c) is an experimental configuration and result, respectively, where the EFIM sensor was revolved. Two dark regions in each circular trace suggest the electric field vector in the direction of white arrow, which is approximately vertical but declines radially off the HDD center.

The case for parallel horizontal scans is shown in Figs. 4(d) and 4(e), where the evanescence of vertical electric field is imaged. The approximate spacing and speed of the scans are respectively $14 \mathrm{~mm}$ and $30 \mathrm{~mm} / \mathrm{s}$, and the imaging time is several tens of seconds. In the image, the evanescent feature appears clearly although an averaging effect and a shift in the imaged distribution are caused by the EFIM sensor size and by the LED lighting asymmetry, respectively. $E_{\mathrm{rms}}$ at the top surface is estimated around $10^{4} \mathrm{~V} / \mathrm{m}$ if $f$ is assumed $50 \mathrm{~Hz}$. As shown in Figs. 4(f) and $4(\mathrm{~g})$, the same scans were applied with a striped sheet of grounded aluminum foil across the top surface. The shielding effect of the foil is thus visualized in Fig. 4(g).

More rigorously, the electric field over the HDD unit was disturbed slightly since the fourth requirement in the above was not sufficiently satisfied. In addition, the frequency dependence of Eq. (1) and Eq. (3) should be taken into consideration if the electric field is spectrally broadened. Downsizing of the EFIM sensor is attractive since the invasiveness and spatial resolution issues as well as the limited frequency band will be improved in inverse proportion to $S$. Nevertheless, it should 
be emphasized that intuition for unseen electric fields can be derived thus promptly by the present method.

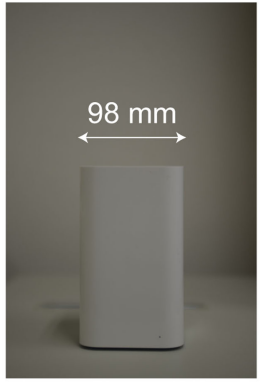

(a)

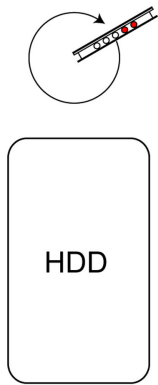

(b)

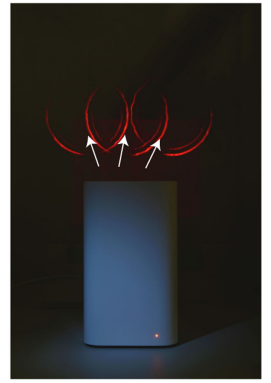

(c)

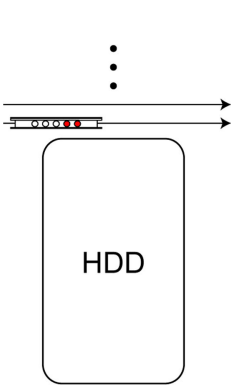

(d)

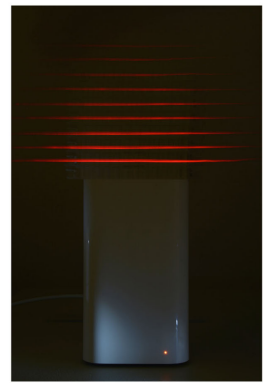

(e)

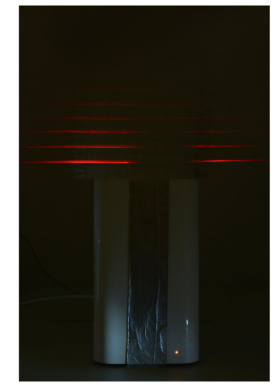

(g)

Fig. 4. Electric fields over an external HDD unit are experimentally visualized by the EFIM sensor. (a) A photograph of the HDD unit. Illustrated in (b) is a revolving scan, which resulted in the photo image in (c), suggesting white arrows as the electric field directions. Horizontal parallel scans in (d) led to indication of evanescent vertical electric field in (e). The same scan set with a stripe of grounded Al foil over the HDD unit in (f) images the shielding effect in $(\mathrm{g})$.

\section{Conclusion}

It should be hereby concluded that the present concept of new electric field sensor has been verified experimentally and its amazing promptness in imaging has been demonstrated successfully. It seems that this concept could pioneer a new sensing technique for electric fields around us, where prompt and familiar visual accesses would be guaranteed even for non-experts.

\section{Acknowledgments}

The authors thank T. Hori for his technical support and M. Onoe for his useful suggestion. Their thanks are due to H. Suzuki, N. Inoue, A. Hara, H. Hara, K. Isaka, and J. Hamasaki for their encouragements. 